\title{
Coronectomy - A viable alternative to prevent inferior alveolar nerve injury
}

\author{
Alok Sagtani ${ }^{1}$, Reshu Agrawal Sagtani ${ }^{2}$, Mehul Jaisani $^{3}$, Leeza Pradhan ${ }^{4}$ \\ 1 Associate Professor, Department of Oral and Maxillofacial Surgery, Nepal Medical College Teaching \\ Hospital, Kathmandu, Nepal. \\ 2 Lecturer, Department of Public Health Dentistry, Nepal Medical College Teaching Hospital, Kathmandu, \\ Nepal. \\ 3 Associate Professor, Department of Oral and Maxillofacial Surgery, BP Koirala Institute of Health \\ Sciences, Dharan, Nepal. \\ 4 Assistant Professor, Patan Academy of Health Sciences, Kathmandu, Nepal
}

Correspondence
Dr. Alok Sagtani
Department of Oral and
Maxillofacial Surgery, Nepal
Medical College Teaching
Hospital, Kathmandu, Nepal.
Email:
drsagtani@hotmail.com

DOI:

http://dx.doi.org/10.3126/ jcmsn.v11i3.14055

\begin{abstract}
Background and Objectives: Coronectomy is a relatively new method to prevent the risk of Inferior Alveolar Nerve (IAN) injury during removal of lower third molars with limited scientific literature among Nepalese patients. Thus, a study was designed to evaluate coronectomy regarding its use, outcomes and complications. Materials and Methods: A descriptive study was conducted from December 2012 to December 2013 among patients attending Department of Oral and Maxillofacial Surgery, College of Dental Sciences, BP Koirala Institute of Health Sciences, Dharan, Nepal for removal of mandibular third molars. After reviewing the radiograph for proximity of third molar to the IAN, coronectomy was advised. A written informed consent was obtained from the patients and coronectomy was performed. Patients were recalled after one week. The outcome measures in the followup visit were primary healing, pain, infection, dry socket, root exposure and IAN injury. The prevalence of IAN proximity of lower third molars and incidence of complications were calculated. Results: A total 300 mandibular third molars were extracted in 278 patients during the study period. Out of 300 impacted mandibular third molar, $41(13.7 \%)$ showed close proximity to inferior alveolar nerve. The incidence of complications and failed procedure was $7.4 \%$ among the patients who underwent coronectomy. During the follow up visit, persistent pain and root exposure was reported while other complications like inferior alveolar nerve injury, dry socket and infection was not experienced by the study patients. Conclusion: With a success rate of $92.6 \%$ among the 41 patients, coronectomy is a viable alternative to conventional total extraction for mandibular third molars who have a higher risk for damage to the inferior alveolar nerve.

Key words: Coronectomy, Inferior Alveolar Nerve Injury.
\end{abstract}

Citation: Sagtani A, Sagtani RA, Jaisani M, Pradhan L. Coronectomy - A viable alternative to prevent inferior alveolar nerve injury. JCMS Nepal. 2015;11(3):1-5.

\section{INTRODUCTION}

Impacted third molars are associated with various pathologies like cysts, tumors, dental caries and pericoronitis. Therefore, total extraction is the appropriate treatment in majority of these cases. However, the surgical procedure is not free of complications. Pain, infection, dry socket, nerve injury and disorders along with their complications are witnessed. ${ }^{1}$ Injury to Inferior Alveolar Nerve
(IAN) during the third molar surgery, entails a sensory deficit that may be temporary (from $0.41 \%$ to $8.1 \%$ ) or permanent (from 0.0145 to $3.6 \%$ ) causing serious problems to the patients. ${ }^{2-4}$ As a result of which various alternatives to total extraction have developed and among them, intentional partial odontectomy (coronectomy) has gained considerable attention in the recent times due to scientific reporting on its benefits. ${ }^{1,5}$ It is 
primarily advised in situations with intimate relationship between the roots of the tooth indicated for extraction (mainly lower third molars) and the IAN, which requires a surgical technique that minimizes the risk of severing, stretching, tearing or compressing the nerve bundle. Thus, a treatment alternative like coronectomy, in which the crown is removed, eliminates the pathology caused by pericoronitis as there is direct closure of the wound, while $\operatorname{root}(\mathrm{s})$ are left behind, preserving the integrity of IAN. ${ }^{5}$

However, there is still speculation about the procedure which does not follow the conventional principle of oral surgery practice which is total extraction. The controversies surrounding this surgical procedure is due to lack of enough scientific evidence regarding long - term outcome of root remnants and possible complications associated with the pulp necrosis. ${ }^{6}$ Along with the procedural problems, there can be possible problems with root remnants such as postoperative pain, dry socket or root migration after certain period of time. ${ }^{7}$ Also, if pulp infection or root eruption takes place, a second surgery is required to complete the extraction. ${ }^{8}$ However, those in favor of the technique indicate that there is less risk of injury to the IAN, as after root migration it is no longer close to the inferior alveolar canal. ${ }^{9}$

Coronectomy is therefore, a relatively new method to prevent the risk of IAN injury during removal of lower third molars with very limited scientific literature regarding its use, outcomes and complications among Nepalese patients. Thus, a study was designed to answer the clinical question of whether coronectomy of lower third molars with close proximity to inferior alveolar canal (indicated on radiographs) can give satisfactory outcomes and can be considered as a good alternative to total removal in Nepalese context.

\section{MATERIALS AND METHODS}

A descriptive study was conducted from December 2012 to December 2013 among the patients attending Department of Oral and Maxillofacial Surgery, College of Dental Sciences, BP Koirala Institute of Health Sciences (BPKIHS), Dharan, Nepal. The inclusion criteria included:

1) Patients presenting with symptomatic unilateral or bilateral impacted mandibular third molars.

2) Radiographic proximity of roots of the tooth to the IAN. The following radiographic criteria was used to decide for proximity of mandibular third molars to inferior alveolar canal:

a) Darkening of the root;

b) Interruption of the white line;

c) Diversion of the canal;

d) Deflection of the root;

e) Narrowing of the root;

f) Narrowing of the canal;

g) Dark and bifid root apex.

The exclusion criteria of the study were:

a) Individuals with history of trauma/surgery to the mandible, developmental anomalies affecting the jaws and clinical and/or radiographic evidence of pathologies of the impacted mandibular third molar teeth which could obscure the visualization of the periapical region or inferior alveolar canal.

b) The patients who refuse to give informed written consent to participate in the study.

All the patients (census sampling) who fulfilled the selection criteria during the study period were included in the study. Clinical examination of the oral cavity was done and panoramic radiographs were made for each study subject using standard exposure and processing protocols. The surgical technique, advantages, and possible complication were explained to the study participants. After reviewing the radiograph for proximity of third molar to the IAN, a single oral and maxillofacial surgeon performed coronectomy on the indicated lower third molar. After performing the surgical procedure, the patients were prescribed antibiotics and analgesics and recalled after one week for suture removal. The outcome measures in the follow-up visit after one week were primary healing, pain, infection, dry socket, root exposure and IAN injury. The patients were advised to contact the department in case of future complications.

The prevalence of IAN proximity of third molars and incidence of complications were calculated. The data was analyzed using Statistical Package for Social Sciences (SPSS) version 20.0. Ethical clearance was obtained from Institutional Ethical Review Board (IERB) of BPKIHS for conducting the study. 


\section{RESULTS}

A total of 278 patients attended the Department of Oral and Maxillofacial surgery, BPKIHS, Nepal during the study duration of one year for extraction of impacted mandibular third molars. In 237 patients, total extraction was performed for 259 impacted third molars which were not close to IAN. Coronectomy was planned and performed among 41 patients who fulfilled the inclusion criteria after panoramic imaging making the prevalence of close proximity of mandibular third molars to inferior alveolar nerve is $13.7 \%$ as shown in fig 1 .

The incidence of complications or failed procedure was $7.4 \%$ among the patients who underwent coronectomy i.e. uneventful primary healing took place among $92.6 \%$ of the patients.(fig. 2)

Of the impacted third molars who underwent coronectomy, one was failed coronectomy i.e. total extraction of mandibular third molar was performed due to peri-operative mobility. During the follow up visit, one patient complained of persistent pain while root exposure was seen in one patient. Reoperation was performed in both the cases. Inferior alveolar nerve injury, dry socket and infection was not seen among the patients. (Fig 3)

\section{DISCUSSION}

A total of 300 mandibular molars among 278 patients were removed in the study period of one year at Department of Oral and Maxillofacial Surgery, BPKIHS. The proportion of molars showing close proximity to inferior alveolar nerve was $13.7 \%$. A profile of patients with IAN lesions found that main causes of inferior alveolar nerve injury were : removal of third molars $(60 \%)$ followed by local anesthesia use (19\%), implant surgery (18\%) and endodontic problems $(8 \%) .{ }^{10}$

Thus, preoperative assessment of proximity of third molars to IAN need to be ascertained with the help of preoperative radiographs. In a setup of eastern Nepal, Computed Tomography imaging is costly for the patients and also the option of Cone Beam Computed Tomography is not locally available, thus, we rely on panoramic imaging. Studies also support that panoramic radiography is still a valuable, cost effective and readily available tool in planning impacted lower third molar surgery to disclose the anatomical relationship with the

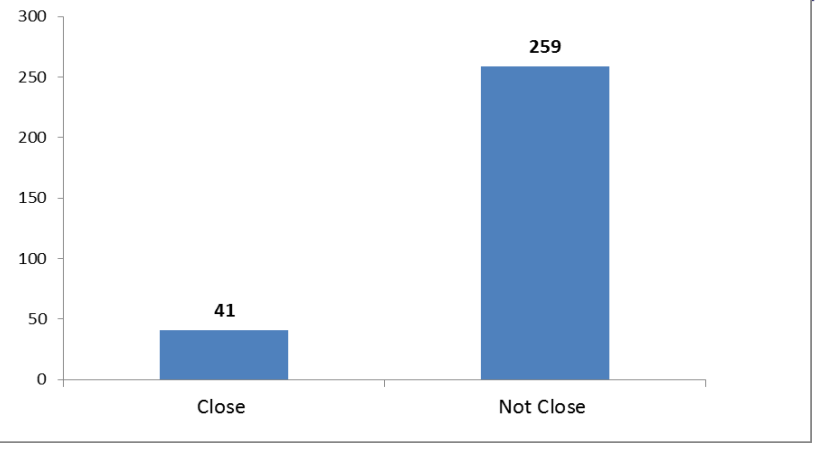

Fig.1: Proportion of mandibular third molars with close proximity to inferior alveolar canal.

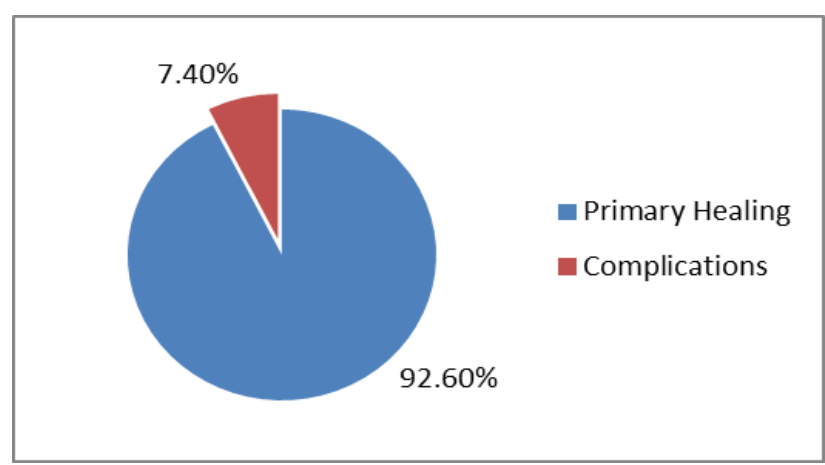

Fig 2: Incidence of complications among patients who underwent coronectomy.

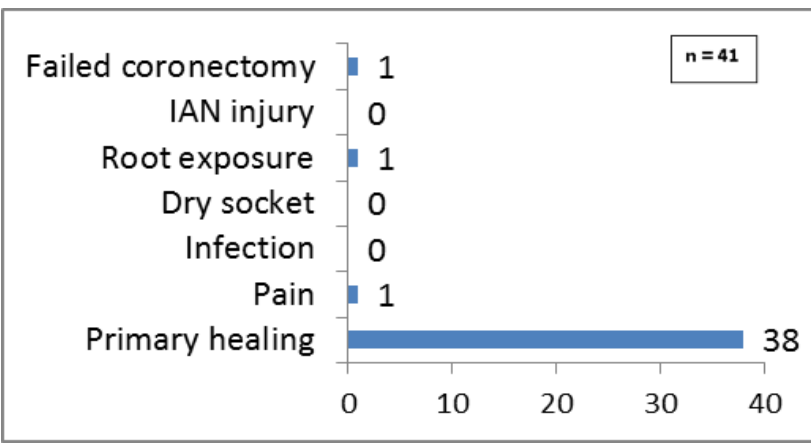

Fig. 3: Outcomes of the coronectomy procedure among patients attending the dental hospital.

Inferior Alveolar Canal. ${ }^{11}$

Among the 41 patients, total extraction had to be performed on $2.4 \%$ of patients due to perioperative mobility. Similar results have been shown in other studies where failed coronectomies due to inadvertent mobilization of remaining root resulted in total extraction in 4 to $38 \%$ of the cases. ${ }^{1,7,12]}$ This can also result in temporary IANI, with an incidence of 8.3 to $11.1 \% \%^{1,12}$ which was not reported by the patients in the present study.

One of the patients complained of pain in the follow -up visit. A randomized clinical trial revealed that in the coronectomy group, one patient complained of pain on the 10th postoperative day and a patient had minor infection 3 to 4 weeks postoperatively. ${ }^{13}$ 
Interestingly, there were no patients showing signs of postoperative infection in the present study. In contrast to this, various clinical trials have published different rates of infection in the coronectomy group. Leung and Cheung ${ }^{[1]}$ reported post operative infection in $5.8 \%$ of patients while Renton reported $3.19 \%{ }^{7}$ and at least $1 \%{ }^{13}$ by this trial.

Root exposure was seen among $2.4 \%$ of the patients in the current study which is high compared to various studies where root exposure rates ranged from 0 to $1.3 \% .^{1,7,14}$

Since, the patients were not followed up for long previous studies have reported root migration among $13.2 \%$ to $85.29 \%$ patients who underwent coronectomy. ${ }^{1,7,14}$ Also, late eruption or root migrations can occur in up to 10 years after coronectomy. ${ }^{15}$

Literature reviews have showed that coronectomy allows migration of the remaining roots away from the path of the nerve, so reducing its possible damage in comparison with total extraction in majority of cases. Also, extraction of the exposed roots would be quite easy and minimize the potential risk of nerve injury. Therefore, root migration and exposure would be beneficial for nerve protection. Thus, concluding that coronectomy as a safe alternative in cases of risk of IAN injury. ${ }^{9,16}$

The current study has few limitations. Firstly, follow-up was done one week after the surgery. This time period is not sufficient to assess long term complications, if any, of coronectomy among the study population. Though, the patients were advised to return for follow up, in case of complications, we cannot be certain about the reason for not coming for follow- up. Secondly, the current study was done among patients of a single dental hospital thus, multi-centric studies with larger sample size need to be carried out to produce robust scientific evidence supporting coronectomy which can be thus, carried out more frequently and with greater confidence among dental practitioners in Nepal.

\section{CONCLUSION}

With a success rate of $92.6 \%$ among the 41 patients, coronectomy is a viable alternative to conventional total extraction for mandibular third molars who have a higher risk for damage to the inferior alveolar nerve among Nepalese patients as well.

ACKNOWLEDGEMENT: We would like to thank all the patients who agreed to participate in the study.

CONFLICT OF INTERESTS: None declared.

\section{REFERENCES}

1. Renton T, Hankins M, Sproate C, McGurk M. A randomised controlled clinical trial to compare the incidence of injury to the inferior alveolar nerve as a result of coronectomy and removal of mandibular third molars. Br K Oral Maxillofac Surg. 2005;43(1):7-12. DOI: 10.1016/j.bjoms.2004.09.002; PMID:15620767.

2. Robinson PP, Loescher AR, Yates JM, Smith KG. Current management of damage to the inferior alveolar and lingual nerves as a result of removal of third molars. Br J Oral Maxillofac Surg. 2004;42:285-92. DOI: 10.1016/j.bjoms.2004.02.024; PMid: 15225944.

3. Renton T. Notes on coronectomy. $\mathrm{Br}$ Dent J. 2012;212:323-6. DOI: $\quad$ 10.1038/sj.bdj.2012.265; PMID:22498530.

4. Gleeson CF, Patel V, Kwok J, Sproat C. Coronectomy practice Paper 1. Technique and trouble-shooting. Br J Oral Maxillofac Surg. 2012;50:739-44. DOI: 10.1016/ j.bjoms.2012.01.001; PMID:22285117.

5. Pogrel AM, Lee JS, Muff DF. Coronectomy: a technique to protect the inferior alveolar nerve. J Oral Maxillofac Surg. 2004;62(12):1447-52 DOI: 10.1016/j.joms.2004.08.003; PMID:15573343.

6. Garcia-Garcia A. Coronectomy: a questionable procedure. J oral Maxillofac Surg. 2005;63(5):723-5. DOI: 10.1016/ j.joms.2005.01.013; PMID:15883954.

7. Leung YY, Cheung LK. Safety of coronectomy versus excision of wisdom teeth: a randomized controlled trial. Oral Surg oral Med Oral Pathol Oral Radiol Endod. 2009; 108(6):

DOI: 10.1016/j.tripleo.2009.07.004; PMID:19782621.

8. Patel V, Gleeson CF, Kwok J, Sproat C. Coronectomy practice. Paper 2: complications and long term management. Br J Oral Maxillofac Surg. 2013;51:347-52. DOI: 10.1016/j.bjoms.2012.06.008; PMID:22794945.

9. Long H, Zhou Y, Liao L, Pyakurel U, Wang Y, Lai W. Coronectomy vs. total removal for third molar extraction: a systematic review. J Dent Res. 2012;91:659-65. DOI10.1177/0022034512449346; PMID:22622663.

10. Renton T, Yilmaz Z. Profiling of patients presenting with posttraumatic neuropathy of the trigeminal never. J Orofac Pain. 2011;25(4): 333-344. PMID:22247929.

11. Mansour S, AlGhamdi AS, Hassanein FAA. Panoramic radiographic signs as predictors of inferior alveolar nerve proximity to the third molar: correlation with surgical and postoperative findings. Egyptian Journal of Oral \& Maxillofacial Surgery. 2012;3(2): 8-85. DOI: 10.1097/01.OMX.0000418703.65978.52.

12. Pogrel AM. An update on coronectomy. Journal of Oral and Maxillofacial Surgery. 2009;67(1):7-12. DOI: 10.1016/j.joms.2009.03.065.

13. Cilasun U, Yildirim T, Guzeldemir E, Pektas ZO. Coronectomy in patients with high risk of inferior alveolar nerve injury diagnosed by computed 
tomography. J Oral Maxillofacial surgery. 2011;69: (6):1557-61. DOI: $\quad 10.1016 /$ j.joms.2010.10.026; PMID:21288616.

14. Hatano Y, Kurita K, Kuroiwa Y, Yuasa H, Ariji E. Clinical evaluations of coronectomy (intentional partial odontectomy) formandibular third molars using dental computed tomography: a case-control study. J Oral Maxillofac Surg. 2009;67(9):1806-14. DOI: 10.1016/ j.joms.2009.04.018; PMID:19686914.

15. Zola MB: Avoiding anesthesia by root retention. J Oral Maxillofac Surg. 1993;51:954 DOI: 10.1016/S0278-2391(10)80128-4.

16. Azorin JFML, Andres GS, MOlina RPV, Muries CA, Panadero RA. Prevention and Treatment of IAN injuries: A literature Review. J Interdiscipl Med Dent SCI. $2014 ; 2: 223$. 